Check for updates

Cite this: J. Mater. Chem. A, 2020, 8 , 15646

Received 22nd April 2020 Accepted 22nd July 2020

DOI: 10.1039/d0ta04181e

rsc.li/materials-a

\section{Direct ink writing advances in multi-material structures for a sustainable future}

\author{
Victoria G. Rocha, (ID *a Eduardo Saiz, ${ }^{\text {b }}$ Iuliia S. Tirichenko (DD b ${ }^{\text {b }}$ and Esther García- \\ Tuñón (iDc
}

Novel manufacturing techniques such as additive manufacturing (AM, also referred to as 3D printing) will play a critical role in building a sustainable future. AM will reduce waste, energy consumption and production time by eliminating the need to assemble components. It will also enable the mass customization of complex devices. To reach their full potential, additive manufacturing technologies should be able to combine different materials in a single processing step. Although the development of multi-material printing is in its infancy, it could have a massive impact in fields as diverse as energy storage and generation, electronic devices, healthcare or structural composites to name a few. Here we provide a critical perspective on the advances and potential of multi-material printing using direct extrusion-based printing, also known as direct ink writing (DIW) or robocasting. We will show examples of devices and structures combining a wide range of materials from ceramics to metals, polymers and carbon with particular focus on three promising applications: energy storage, lightweight composites and sensors. The goals are to assess the progress made so far, to point out specific challenges and areas for further development and to provide guidelines to those interested in multi-material DIW.

\section{Introduction}

${ }^{a}$ School of Engineering, Cardiff University, Cardiff CF243AA, UK. E-mail: GarciaRochaV@cardiff.ac.uk

${ }^{b}$ Centre for Advanced Structural Ceramics, Imperial College London, London $S W 72 A Z$, UK

'School of Engineering, Materials Innovation Factory, University of Liverpool, UK

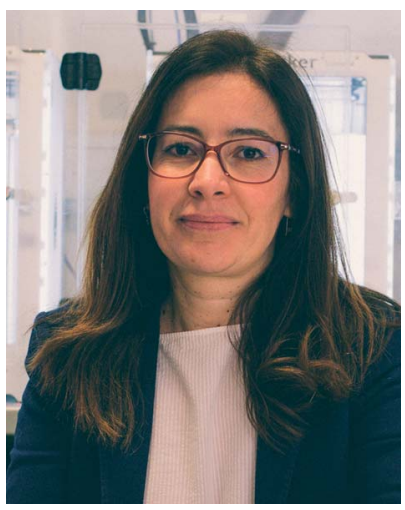

Victoria G. Rocha is Honorary Research Fellow in the School of Engineering at Cardiff University and has been recently awarded a Ramón y Cajal Tenure Track fellowship to join the National Institute of Carbon Science and Technology, CSIC, in Spain where she had obtained her PhD in Materials Science in 2008. Before joining Cardiff University, she worked at ITMA Materials Technology, Bath University and she was Intra-European Marie Curie Fellow at the Centre of Advanced Structural Ceramics at Imperial College London. Her research focuses on design and formulation of multimaterial pastes for extrusion-based printing in energy storage applications and composites but also on processing and development of novel bioinspired graphene/ceramic composites through spark plasma sintering.
In 2015 the United Nations agreed the 2030 Agenda for Sustainable Development. Goals 9 and 13 mention the importance of sustainable industrial development, and of taking actions to combat climate change respectively. ${ }^{1}$ To achieve

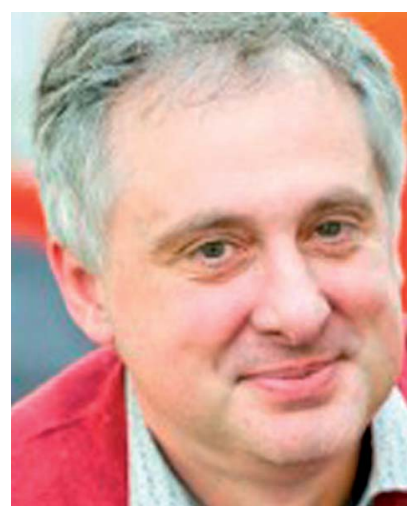

Eduardo Saiz is a Professor in the Department of Materials at Imperial College, where he directs the Centre for Advanced Structural Ceramics. He received his $P h D$ degree in Applied Physics from Universidad Autónoma de Madrid. Before joining Imperial College, he worked at Lawrence Berkeley National Laboratory. His research focuses on the processing and characterization of ceramics and ceramic-based composites for different applications from aerospace to healthcare and the study of high temperature interfacial phenomena. 
those goals industry must implement innovative approaches to manufacture more efficiently with less raw materials in order to build a future that is more prosperous, more just, and more secure. Traditional mass production of identical commodity items usually involves processes such as casting, forging or machining, which do not minimise waste. At the same time many of these processes limit the complexity of the structures that can be fabricated and therefore impose additional design constraints. Many efforts are being made to develop novel manufacturing techniques such as additive manufacturing (AM, also referred to as $3 \mathrm{D}$ printing) that unlike casting do not rely on the use of moulds and enable the fabrication of complex geometric shapes that can be mass-customized. AM is a group of technologies capable to build structures layer-by-layer through a series of cross-sectional slices, that are generated by a design software. AM design concepts are translated into products via digital manufacturing which allows merging of multiple components in one fabrication step without additional assembly operations, thus saving materials and energy. ${ }^{2}$ There are over fifty different AM techniques usually classified into seven different processes in accordance to the ASTM F2792-12a: binder jetting, material jetting, material extrusion, vat photopolymerisation, powder bed fusion, energy deposition and sheet lamination. ${ }^{3}$

The published literature on AM has grown exponentially since 2004, being major contributors to its development scientists and engineers from a wide range of disciplines from material science to chemistry and physics, mechanical engineering and artificial intelligence. The outcomes of this research investment are having an impact in numerous fields from healthcare, electronics and civil engineering to energy generation and storage, environmental engineering, education or entertainment to name some. ${ }^{2}$ As this emerging field quickly develops, the number of published reviews in AM doubles every two years. Some of them focus on objects made of an individual family of materials such as polymers, ${ }^{4,5}$ metals, ${ }^{6-8}$ ceramics, ${ }^{9}$ carbon-based $^{\mathbf{1 0}}$ materials and even nanomaterials ${ }^{\mathbf{1 1}}$ using different AM techniques, while others cover a single material or a very specific technique. The number of reviews focusing on a specific application such as energy storage ${ }^{12-16}$ and electrochemical devices ${ }^{17-19}$ is also growing, which reveals the fast development of a wide multi-disciplinary field. A review by MacDonald et al. ${ }^{\mathbf{2 0}}$ highlights the potential of multi-process (or hybrid) 3D printing, in which complementary technologies, both novel and traditional, are 'collaboratively' combined to advance the future of manufacturing. The authors state that the next generation of $3 \mathrm{D}$ printing will enable the integration of dissimilar materials and allow the integration of active components to deliver functionality that was not possible previously. ${ }^{20}$

The development of multi-material printing is at its infancy however it could have a massive impact for example in energy applications, electronic devices and composites. Combining different materials in a single manufacturing process will reduce waste and production time by eliminating the need to assemble components. It will also expand the design space, enabling new products, composites and devices with structural and functional properties. Among all the AM processes only material jetting and material extrusion have shown promising results in multi-material printing, one of the biggest challenges in AM today.

Material extrusion techniques such as fused deposition modelling (FDM) enable multi-material structures by melting filaments made of pure polymeric materials or a combination of different polymers with functional materials. Several reviews ${ }^{\mathbf{4 , 5}}$ have highlighted the success. Here we provide a critical perspective on the fabrication of 'truly' multi-material structures in which different classes of materials, such as ceramics, metals, polymers and carbon-based materials are combined using direct ink writing (DIW) 3D printing.

3D-printing through material extrusion of viscoplastic pastes (also referred to as 'inks') is also known as direct ink writing

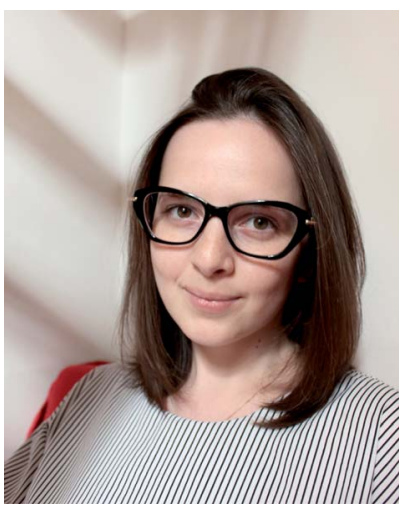

Iuliia S. Tirichenko is currently a postdoctoral research associate in the Centre for Advanced Structural Ceramics (CASC), Department of Materials, Imperial College London. She received her specialist degree in Physics of Metals from Voronezh State Technical University, Voronezh, Russia and her PhD in Chemical Engineering from Imperial College London. Her research interests include colloid science, processing, and formulations for additive manufacturing of ceramic materials.

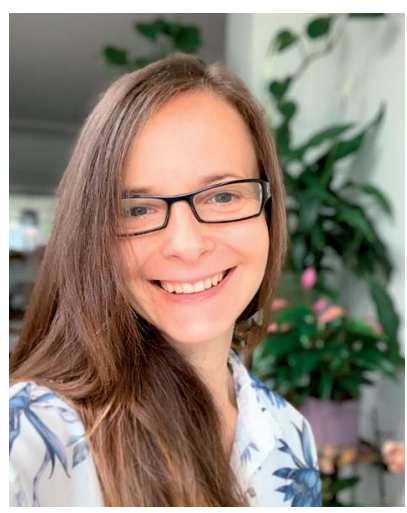

Esther García-Tuñón completed her degree in Chemical Engineering and $a \mathrm{PhD}$ in Materials Science from the University of Santiago de Compostela, Galicia (Spain). She moved to the UK to join the Centre for Advanced Structural Ceramics (CASC) at Imperial College London with a Barrie de la Maza fellowship in October 2011. After five years as a postdoctoral researcher at Imperial, Esther joined the University of Liverpool in 2017 as a Lecturer in Materials Science and Engineering. Her group, affiliated to the Materials Innovation Factory (MIF) and the School of Engineering, works across different disciplines. Her research interests include processing, complex fluids and rheology. 
(DIW) or robocasting. DIW was initially developed to create complex ceramic structures, hence the term robocasting, and so far, has been mainly exploited in research labs for small scale fabrication. The number of research groups using this technique has expanded worldwide beyond the structural ceramics field into other areas, such as 3D bioprinting, ${ }^{21-23}$ energy, ${ }^{24-27}$ composites, ${ }^{28,29}$ sensors, ${ }^{30}$ robots $^{31}$ etc. DIW is the most versatile $\mathrm{AM}$ technique in terms of materials development, it enables the creation of complex 3D shapes using any material by formulating a paste with controlled rheology ${ }^{27,32-36}$ (a shear-thinning yield stress fluid ${ }^{37}$ ). The current advances in formulation $\operatorname{design}^{34,38}$ and the fundamental understanding of the rheological parameters that define 'printability ${ }^{\mathbf{2 9 , 3 5 , 3 9 , 4 0}}$ will pave the way for scaling up and industrial use. Another advantage of DIW is that it enables a combination of different formulations into complex structures by using multiple extrusion nozzles (Fig. 1). Modifications of the extrusion system by using microfluidic printheads allows to switch ${ }^{\mathbf{4 1 , 4 2}}$ or $\operatorname{mix}^{\mathbf{4 3 , 4 4}}$ (Fig. 1) different materials in a single pass. Multi-material DIW has also the potential to provide engineered interfaces. Formulations with similar composition and rheology have been combined to make soft structures, ${ }^{42}$ or glass with varying compositions. ${ }^{45}$ However, so far there are only a few examples in the literature of what we

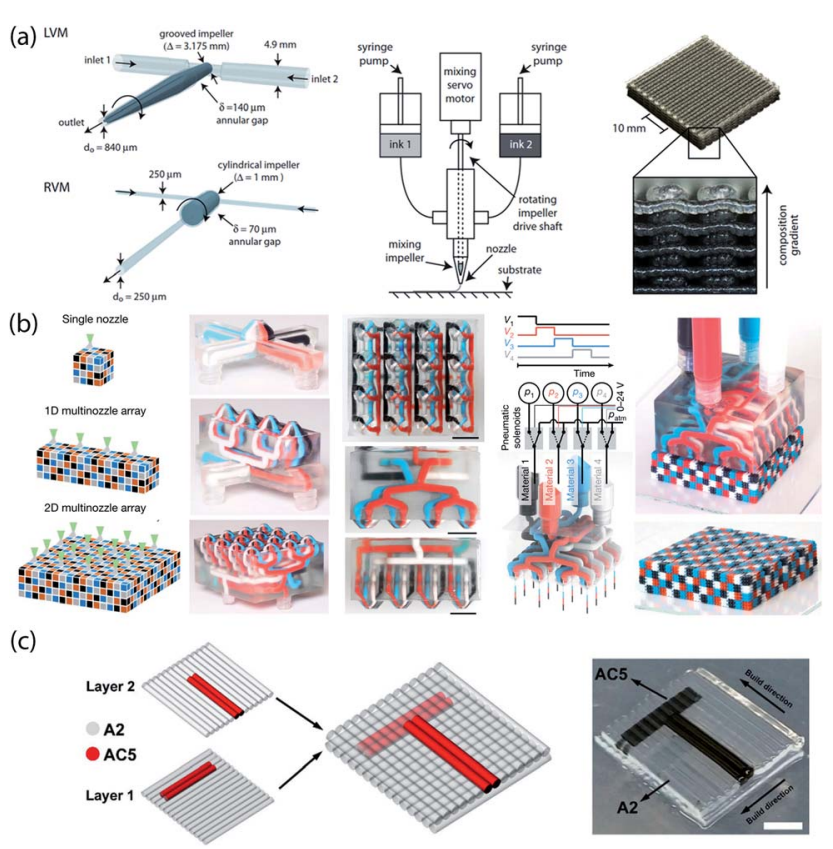

Fig. 1 Multi-material 3D printing approaches for soft-materials. (a) Systems for active mixing in situ during printing using rotating impellers (grey shadow) designed for large (LVM) and reduced (RVM) volumes. These systems enable the integration of multi-material graded compositions into a structure. Reproduced from ref. 44 with permission from John Wiley and Sons. (b) Multi-material, multi-nozzle 3D printheads in a microfluidic system combining fast pneumatic solenoids and soft inks enable voxelated printing. Reproduced from ref. 42 with permission from Springer Nature. (c) Multi-material deposition of hydrogel structures based on an aspiration-on-demand protocol. The constitutive multi-material segments of extruded filaments were first assembled in liquid state by sequential aspiration of inks into a glass capillary, followed by in situ gel formation. Scale bar (2 $\mathrm{mm}$ ). Reproduced from ref. 46 with permission from Springer Nature. consider 'truly' multi-material structures. In part this is due to the limitations imposed by the post-printing steps (drying, debinding and consolidation) that are often necessary but restrict the combination of materials with very different properties (e.g. thermal expansion, melting point or oxygen sensitivity). An alternative approach is to formulate printable materials that can be shaped and used as printed, avoiding post-processing without compromising functionality. This approach has been used to make thick semisolid electrodes for Li-ion batteries. ${ }^{26}$

In this perspective, we review the progress made to date pointing out specific challenges and areas for further development in order to provide guidelines for those interested in multi-material DIW. We highlight successful examples in three promising applications: energy storage, lightweight composites and sensors.

\section{Direct ink writing for multi-material printing}

Current approaches for direct ink writing of disparate materials with high spatial resolution are based on precision motion control systems. This is normally an XYZ gantry robot with a few microns $(1-3 \mu \mathrm{m})$ accuracy and repeatability in the 3 directions. At the laboratory scale, this robot is normally equipped with linear motors that can reach maximum speeds between 0.1 and $2 \mathrm{~m} \mathrm{~s}^{-1}$. The gantry robot is coupled with a printer head device with multiple syringes pumps or pressure-driven reservoirs for different formulations. Disparate materials can be combined in the same object by synchronising XYZ motion with the plunger displacement or applied pressure. The precise movements of the XYZ gantry robot and syringe pumps are controlled through a user interface or software that translates a predefined $3 \mathrm{D}$ design into g-code commands that guide the printing of the desired object.

Several add-ons and modifications of the basic set-up described above can expand the versatility of the technique and the range of formulations used to increase the complexity of the printed object. For example by designing active mixers ${ }^{43}$ and droplet generators; ${ }^{47}$ attaching CCD cameras ${ }^{48}$ or UV-light sources to cure polymers ${ }^{49}$ (Fig. 2); using humidifiers, suspension baths for embedded printing, and heated syringes and/or platforms; ;0 $^{50 m b i n i n g ~ D I W ~ w i t h ~ a d d i t i o n a l ~ F u s e d ~ D e p o s i t i o n ~}$ Modelling (FDM) printer heads ${ }^{51}$ (Fig. 2); or simply by placing the system under controlled atmospheres. ${ }^{26}$

The main difference between FDM and DIW is that in FDM polymeric filaments must be melted during extrusion, while DIW systems rely purely on pastes with very specific rheology that are stored in a syringe cartridge. These complex fluids are formulations that typically comprise a mixture of powdered materials (and in most cases different additives) in a liquid with concentrations that are systematically adjusted to achieve a paste with yield stress and shear thinning behaviour. After careful mixing and conditioning (e.g. defoaming, cooling or heating, or even after reactions that may need to take place between the components in the mixture) these pastes should 
(a)

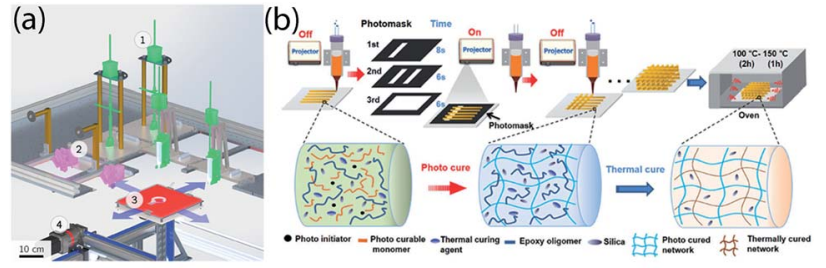

(c)

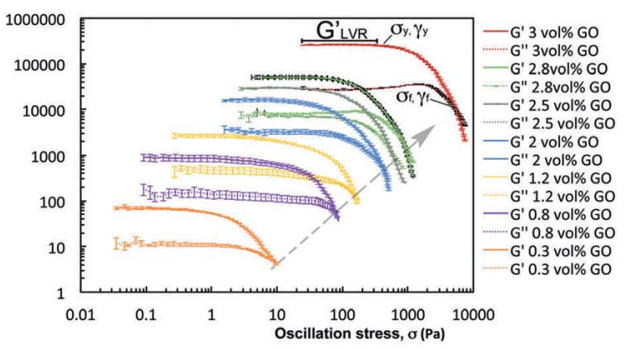

(d)

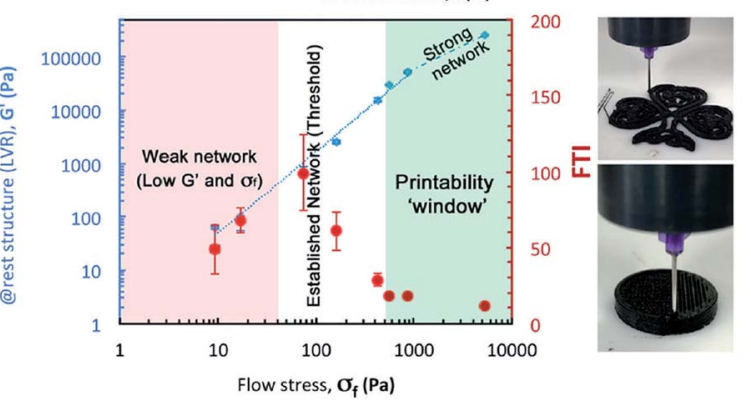

Fig. 2 Examples of modified DIW systems ( $a$ and $b$ ) and criteria for ink printability ( $c$ and $d$ ). (a) The scheme shows a multi-phase additive manufacturing system that combines DIW components (1) with FDM modules (2) heated platform (3) and with gear-boxes allowing spatial resolution of $\pm 3.4 \mu \mathrm{m}$ (4). Reproduced from ref. 51 with permission from Elsevier. (b) Schematic showing the processes involved in a dynamic photomask-assisted DIW multi-material system. The DIWprinted structure is placed under the dynamic photomask which consists on a projector with a set of light patterns whose display time is controlled by the software. Reproduced from ref. 52 with permission from John Wiley and Sons. (c) Amplitude sweep at fixed frequency of inks with varying graphene oxide (GO) content showing some of the main rheology parameters $\left(G^{\prime}, G^{\prime \prime}\right.$ and flow stress) that can be used to quantify printability. Reproduced from ref. 35 with permission from RSC. (d) Printability map that shows the optimum region for DIW of graphene oxide suspensions, those with a crossover point (flow stress) above $500 \mathrm{~Pa}, G_{\mathrm{LVR}}^{\prime}$ of the order of $10^{4}$ to $10^{5} \mathrm{~Pa}$, and FTI below 20 with very small uncertainties. Reproduced from ref. 35 with permission from RSC.

easily flow under shear and quickly recover upon deposition. Printable formulations are non-Newtonian fluids with a yield stress often described as Herschel-Bulkley fluids $\left(\tau=\tau_{\mathrm{y}}+K \dot{\gamma}^{n}\right.$ where $\tau(\mathrm{Pa})$ is the shear stress applied, $\tau_{\mathrm{y}}(\mathrm{Pa})$ is the yield stress, $K\left(\right.$ Pa s$\left.^{n}\right)$ the viscosity parameter and $\dot{\gamma}\left(\mathrm{s}^{-1}\right)$ the shear rate). ${ }^{32}$ Other models for non-Newtonian fluids with a yield stress could be more suitable but they have not been widely used in the DIW community so far. ${ }^{53}$ In general, beyond the yielding point these yield stress fluids have a shear thinning behaviour with very small flow index values (the dimensionless coefficient $n$ that measures to which degree the fluid is shear thinning or shear thickening, for Newtonian fluids $n=1$ ). Shear tests for printable pastes are extremely affected by transient effects, shear banding and shear induced fracture so data interpretation and analysis should be carefully done. After the yielding point the flow index can be more accurately determined from a viscosity plot $\left(\log \mu\right.$ (Pa s) vs. $\left.\log \dot{\gamma}\left(\mathrm{s}^{-1}\right)\right)$ using the power law model $\tau=K \dot{\gamma}^{n}$ and only with the data in the shear thinning region at mid-shear rates. Oscillatory rheology is a less uncertain alternative to quantify printability using bulk rheological parameters obtained from an amplitude sweep: the storage modulus in the linear viscoelastic region (LVR), $G_{\mathrm{LVR}}^{\prime}(\mathrm{Pa})$; the crossover point ( $\mathrm{Pa}$, stress at which $G^{\prime}=G^{\prime \prime}$, where $G^{\prime \prime}$ is the loss modulus); and the flow transition index, FTI (dimensionless, stress at yielding point $\tau_{\mathrm{yp}}\left(G^{\prime}=0.95 G_{\mathrm{LVR}}^{\prime}\right) /$ stress at the crossover, $\left.\left(G^{\prime}=G^{\prime \prime}\right)\right)$ (Fig. 2c and d). ${ }^{35}$ The figure of merit (FoM, $G_{\mathrm{LVR}}^{\prime}$ /stress at crossover) is another dimensionless number that can provide guidance in formulation design. ${ }^{29}$ As a benchmark guide the crossover stress $\left(G^{\prime}=G^{\prime \prime}\right)$ determined from an amplitude sweep must be high enough for the formulation to be self-supportive in the absence of stress $(\sim 500 \mathrm{~Pa})$ but not too high $(<2500 \mathrm{~Pa})$ as this could easily compromise the extrusion process by clogging the nozzles or leading to filter press effects for filled systems. The kinetics of the recovery scales with $G_{\mathrm{LVR}}^{\prime},{ }^{35}$ which must broadly reach values of the order $10^{4}$ to $10^{5} \mathrm{~Pa}$ for DIW applications but heading towards the upper range $\left(10^{5} \mathrm{~Pa}\right)$ to guarantee good printing resolution of multi-material structures. Formulations should also have FTI values below 20 and FoM values above 20 . The wider DIW community will benefit from adopting a systematic rheological characterisation using common protocols and defining the meaning and origin of every rheological parameter used. For example, the cross over point $\left(G^{\prime}=\right.$ $G^{\prime \prime}$ ) can be referred as the yield stress or flow stress depending on the source, while some papers refer only to viscosity values at a certain shear rate, which is not enough to characterise such complex fluids.

\section{DIW approaches to multi-material structures: challenges and opportunities}

Different approaches can be used to print multi-material structures, each of them with potential in different fields but also with specific technical issues. Here we classify them based on the number of printing cartridges used:

-Single-paste cartridge. In these systems, an object is constructed using a multi-material ink extruded from a single cartridge through a single nozzle. We include here those systems in which the composition of the ink may be gradually changed (e.g. by using in situ mixing to change the ratio between two components). A variant of multi-material building using a single cartridge is the use of robotic-assisted deposition to build preforms that are then filled by a second phase.

-Multiple-pastes cartridges. In these systems, an object or a device is built by alternatively extruding formulations with different compositions from different cartridges through one to multiple nozzles. It involves switching of cartridges and/or nozzles on the flight during printing. This is the approach 
generally considered as "true" multi-material printing that has been used to print complex devices like those designed for energy storage.

-Co-extrusion of pastes. Between the two approaches described above are coextrusion systems designed with concentric nozzles to print core-shell filaments (with the core and shell from different compositions). There are already largescale co-extrusion systems designed to print polymer-based composites.

This simple classification enables us to highlight specific challenges and areas for development. In the next subsections, we review specific examples for each approach.

\subsection{Single-paste cartridge systems}

A wide range of multi-material inks have been formulated to print composites for different applications, from lightweight structural materials, ${ }^{28,54}$ to tissue engineering scaffolds, ${ }^{55}$ sensors $^{30,56-59}$ or thermoelectric devices. ${ }^{60}$ All these applications demand careful structural control not only at the macrolevel (where 3D printing has clear advantages) but also at the micro level and below. The later has been a challenge but as the technology advances it is also becoming evident that the extrusion process itself can be used to open new possibilities in microstructural design. Several groups have used additive manufacturing to create porous preforms that are infiltrated with a second phase. ${ }^{61}$ In this case, the preform design is used to control microstructure. Another fruitful approach is the use of flow during extrusion, sometimes in coordination with external fields, to align anisotropic particles (typically fibres or platelets) in a matrix (usually organic). ${ }^{62,63}$ In some cases, both approaches are combined and preforms are built using fibres or platelets that can be subsequently infiltrated to generate the composite material. ${ }^{29}$

Usually, when printing structural composites, the focus is often to enhance fracture resistance. By controlling the distribution and orientation of stiff and anisotropic reinforcements in a matrix it is possible to manipulate crack paths and promote extrinsic toughening. This opens new opportunities for ceramic-based composites that are often the only alternative for applications at high temperature or in highly corrosive environments and whose use is in many cases limited by their fracture resistance rather than their strength. Printed composites have also been used in sensors for stress or temperature developed for soft robotics ${ }^{64}$ or wearable devices. ${ }^{65}$ Inks containing carbon nanotubes or graphene enable the formation of carbon networks in elastomeric polymeric matrices that can be used for piezopotential or resistive sensing. An illustration in the case of resistive sensing is the alignment during printing of graphene sheets in a PDMS matrix that results in interconnected networks with enhanced electrical conductivity at lower carbon contents. As a result, variations of electrical resistance with flexion or pressure can be easily detected. Carbon nanotubes networks in a PDMS matrix also enhance the performance of printed piezoresistive strain sensors. ${ }^{\mathbf{6 6}}$

Another area of application is in the field of active materials: $4 \mathrm{D}$ printing. The fourth dimension is described by Tibbits ${ }^{67}$ as transformation over time, emphasising that printed structures are no longer simply static, dead objects; rather, they can be programmed to be active and can transform independently. DIW single-cartridge systems have been successfully used to print soft material composites whose microstructure is designed to provide programmable shape response (after immersion in water, ${ }^{68}$ using magnetic fields ${ }^{69}$ or temperature $\mathrm{control}^{\mathbf{7 0}}$ ) yielding complex three-dimensional morphologies.

In general, for extrusion-based additive manufacturing to provide further practical advances it will be necessary to push the limits of structural manipulation at all length scales. This type of control can often be achieved by manipulating the composition of the inks and the size and structure of the particles used in their formulation. For instance, work with

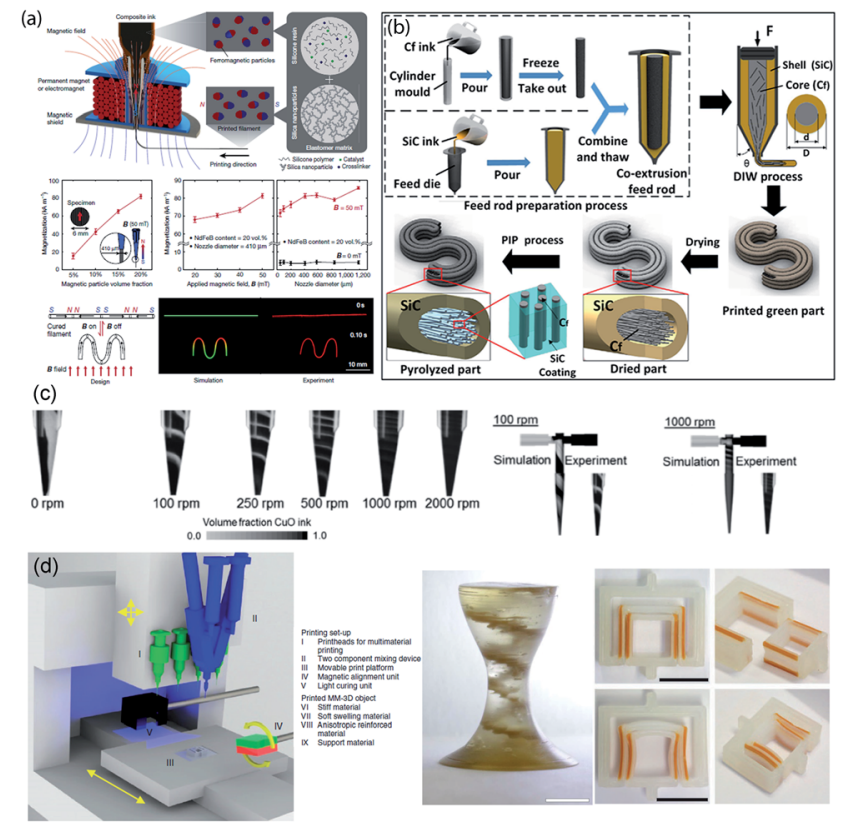

Fig. 3 Multi-material structures using single-cartridge (a), co-extrusion (b), active mixing (c) and multi-cartridge (d) DIW approaches. (a) Example of 4D printing using a single cartridge: $\mathrm{NdFeB}$ ferromagnetic particles embedded in an elastomeric composite paste can be aligned on demand through a magnetic field coupled to the DIW nozzle to fabricate shape-programmable soft materials (4D printing). Reproduced from ref. 69 with permission from Springer Nature. (b) DIW coextrusion methodology of a core-shell carbon fibers/silicon carbide $\left(\mathrm{C}_{f} / \mathrm{SiC}\right)$ lightweight composite. The dense $\mathrm{SiC}$ shell provides strength while the core contains highly oriented $\mathrm{C}_{\mathrm{f}}$ that enhance the material toughness. Reproduced from ref. 75 with permission from Elsevier. (c) Combination of an active mixer with DIW to control spatially the extent of a thermite reaction ( $\mathrm{Al}, \mathrm{CuO}$ ) within a structure. The speed of the rotating impeller gives an additional degree of control over the mixing and results in a homogeneous composition at sufficiently high rotational speeds. This is a promising pathway for the manipulation of microstructure within a printed object. Reproduced from ref. 74 with permission from John Wiley and Sons. (d) Multi-cartridge system with add-ons, including a magnetic field generator, an UV-light for in situ curing and movable platform. An ink of magnetically responsive anisotropic and stiff particles was printed to make an helicoidal staircase suspended in a light-sensitive liquid resin of tuneable composition. Soft mechanical fasteners fabricated through programmable multi-material magnetically assisted DIW printing of shape-changing objects. Printed key-lock objects before and after the shape change. Reproduced from ref. 63 with permission from Springer Nature. 
platelet-based materials suggests that platelet roughness at a submicron level can have a large contribution to the mechanical response of composites. ${ }^{71,72}$ It can be more difficult to manipulate structure at the microlevel and this may require modifications of the printing system as in the use of magnetic fields to manipulate particle orientation ${ }^{63}$ or systems designed to actively $\operatorname{mix}^{\mathbf{4 3 4 4}}$ the cartridge in situ during printing while controlling the feeding of the different components to enable compositional variations along the structure ${ }^{73,74}$ (Fig. 3).

\subsection{Coextrusion of pastes}

Co-axial extrusion systems have been used to print ceramic matrix composites ${ }^{75}$ filaments with sacrificial cores that are subsequently eliminated to create channels ${ }^{76}$ and cellular structures with high stiffness built from filaments combining stiff, brittle epoxy shells with a flexible core (Fig. 3b). ${ }^{77,78}$ These designs are often limited by the diameter of the co-axial printing nozzles. Composite manufacturing will also demand the development of effective strategies to engineer the core-shell interface for a wide range of material combinations.

\subsection{Multi-paste cartridge systems}

Most advances in this area have been made to create devices for electrochemical energy storage. This is one of the fields that is pushing the multi-material challenge given the nature of the application, in which the combination of very disparate materials from polymers, ceramics and metals in one single device is key for performance (Fig. 4).

When printing using multiple nozzles, in addition to adjusting the ink rheology to satisfy printing demands, there are other technical aspects that must be considered like tip alignment and start/stop flow settings. Tip alignment is crucial to guarantee precise control of the interface between the extruded lines, which may become even more challenging as the number of cartridges increases. Start/stop flow settings mainly depend on the density and rheology of the formulation. When switching from one nozzle to the next one, some inks may continue flowing when the plunger stops moving, making necessary to pull back the plunger to stop the flow. The "extra" flow of material from the tip is likely related to gravitational and rheological effects as inertial effects can be dismissed based on the very low Reynolds number $(\operatorname{Re}=\rho v D / \mu$ where: $\rho$ is the density $\left(\mathrm{kg} \mathrm{m}^{-3}\right), v$, the velocity $\left(\mathrm{m} \mathrm{s}^{-1}\right), D$, the diameter of the nozzle (m) and $\mu$ the viscosity (Pa s)) values for highly viscoelastic shear thinning fluids flowing through very small nozzles. For example, Re values are of the order of $10^{-3}$ to $10^{-5}$ for graphene oxide-based inks at estimated shear rates of $20 \mathrm{~s}^{-1}$ and $200 \mathrm{~s}^{-1}$ in the nozzle, and printing speeds of $10 \mathrm{~mm} \mathrm{~s}^{-1}$. To effectively stop flow during switching, the back-off distance setting brings the plunger back and pulls the material inside the syringe preventing ink overflow, this distance must then be accounted for when pushing the plunger down to re-initiate flow. An added difficulty is that each DIW system may have different processes to ensure tip alignment, define and code printing settings to minimize flaws when switching cartridges, which often are not detailed in published literature.

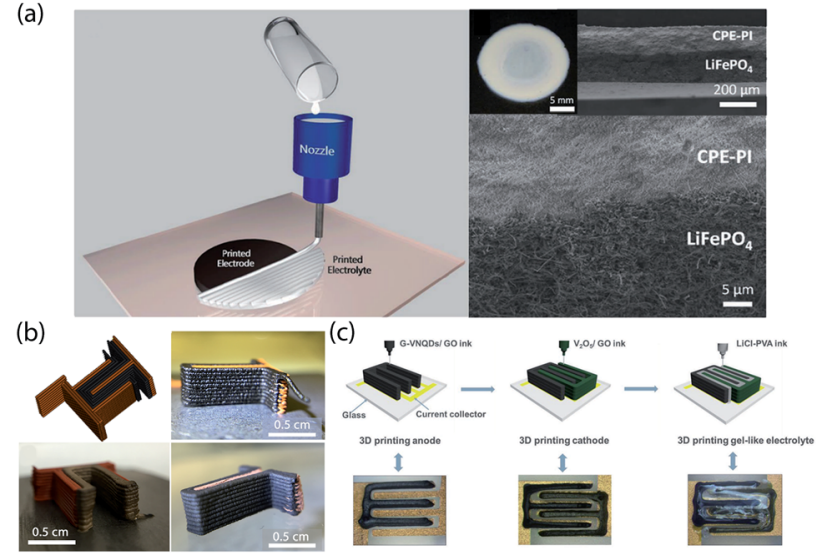

(d)

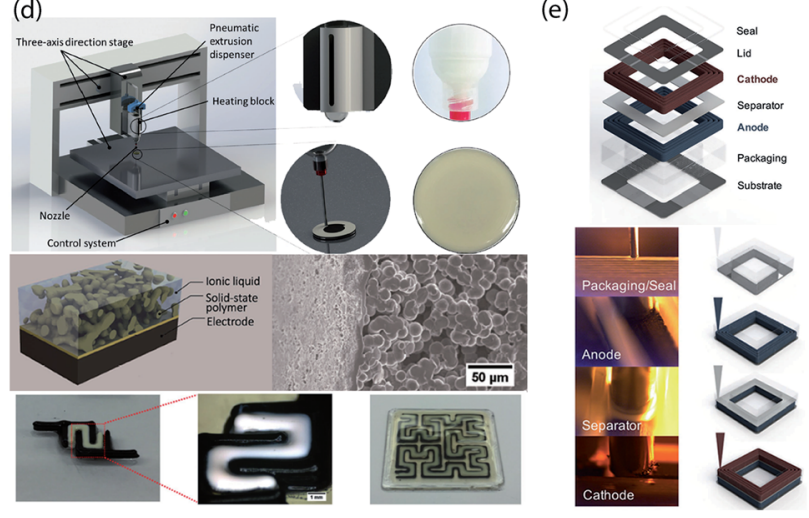

Fig. 4 Multi-material structures for energy applications using multicartridge DIW approaches. (a) The ink formulations used to fabricate a polymer electrolyte and a carbon/ceramic electrode via sequential printing led to a tight and continuous interface between the electrode and electrolyte layers. The electrochemical performance of these flexible devices is on par with commercial systems even at current rates as high as $5 \mathrm{C}$. Reproduced from ref. 24 with permission from John Wiley and Sons. (b) An interdigitated supercapacitor made of reduced graphene oxide and copper was fabricated by combining Pluronic-F127®-based inks to formulate both the electrode and current collector pastes, multi-cartridge DIW and post-printing heat treatments. Reproduced from ref. 27 with permission from American Chemical Society. (c) Complexity via interdigitated multi-material DIW printing was also achieved for an asymmetric micro supercapacitor (MSC). The combination of an ink formulation based on graphene oxide as binder for the electrodes and a gel-electrolyte with several post-printing steps (freeze drying and hydrazine chemical reduction) resulted in a complex-shaped device with ultrahigh areal energy density and excellent performance. Reproduced from ref. 79 with permission from John Wiley and Sons. (d) Proof of concept of complex Li-ion batteries with solid electrolytes via multi-cartridge DIW: the inks must be formulated to reach good interfacial contact and electrochemical performance. This was first achieved on a flat system using $\alpha-\mathrm{MnO}_{2}$ as electrode, then a heated-cartridge add-on allowed extrusion of a solid electrolyte paste. Finally, a proof of concept of an interdigitated Li-ion battery was DIW using a LTO/solid electrolyte/LFP and PDMS as packaging. Reproduced from ref. 50 with permission from John Wiley and Sons. (e) Fully 3D printed and packaged Li-ion battery (LIB) made using multi-cartridge DIW. The post-processing steps were eliminated through careful formulation design, placing the printer in an Ar-filled glove box and using UV light source to cure the packaging in situ. Reproduced from ref. 26 with permission from John Wiley and Sons. 
One of the earliest successful attempts at multi-material DIW was the printing of the most fundamental components of a $\mathrm{Zn}$-air battery, ${ }^{\mathbf{8 0}}$ including the $\mathrm{Zn}$ anode, separator, cathode catalyst, anode/cathode terminals, and casing. The main challenge in this work was to select material formulations that could be deposited to fabricate all the essential components in the battery without compromising the performance of the final device. Subsequently, a huge effort has been made in the field to develop inks/pastes for each component and a large number of reviews on 3D printing for energy storage have been published but there are still very few examples in which multi-material devices have been successfully fabricated. So far, there have been many attempts to print electrodes and some solid electrolytes. ${ }^{\mathbf{1 4 , 8 1}}$

One of the challenges in multi-cartridge printing is to engineer the interfaces between the different components to achieve the right degree of interconnection. Several research groups are trying to develop universal ink formulations based in the same carriers for different materials to ensure good interfacial contact and, if possible, avoid post-processing after printing. For example, Durstock et $a .^{24}$ used a dry inversion method and a nanosized alumina filler to control the porosity of the polymeric electrolyte (Fig. 4a). They used a similar approach for the carbon/ ceramic electrode formulation and thus succeed in DIW discs of both electrode and electrolyte while maintaining expected functionalities in both layers. This formulation approach demonstrates that the electrolyte can be sequentially printed directly over an electrode layer without sacrificing performance. They achieved a tight and continuous interface, which is a desired trait for discharge voltage stability in a flexible energy storage device under mechanical load. Similarly, Rocha et $a .^{27}$ successfully printed a symmetric supercapacitor device by designing an environmentally friendly formulation that includes a thermoresponsive polymer as a carrier of both graphene oxide (electrodes) and copper (current collector) (Fig. 4b). This work illustrates another challenge. Often post-printing heat treatments are required to dry and eliminate organics as well as to consolidate the parts. These treatments should be compatible with all the materials allowing an accurate control of dimensional changes and the formation of stable interfaces. In this case, both graphene oxide reduction and $\mathrm{Cu}$ sintering can occur at the same temperature and atmosphere enabling the fabrication of an interdigitated shape with a strong interface between the current collector and the binder-free electrode.

Recently Yang et $a .^{79}$ used DIW to make a multi-material asymmetric micro supercapacitor (MSC) with ultrahigh areal energy density (Fig. 4c). Cathode and anode inks of vanadium pentoxide $\left(\mathrm{V}_{2} \mathrm{O}_{5}\right)$ and graphene-vanadium nitride quantum dots (G-VNQDs) with highly concentrated graphene oxide (GO) dispersions, as well as a LiCl-PVA gel electrolyte were designed and formulated. The 3D printed asymmetric MSC with interdigitated electrodes exhibited excellent structural integrity, a large areal mass loading of $3.1 \mathrm{mg} \mathrm{cm}^{-2}$, and a wide electrochemical potential window of $1.6 \mathrm{~V}$. Moreover, there were substantial open macropores in the 3D printed electrodes, which provided numerous channels to facilitate mass transportation.
Cheng et al. ${ }^{50}$ also tackled the challenge of adding complexity and printed an interdigitated full cell battery. Lithium iron phosphate (LFP) and lithium titanium oxide (LTO) materials

(a)

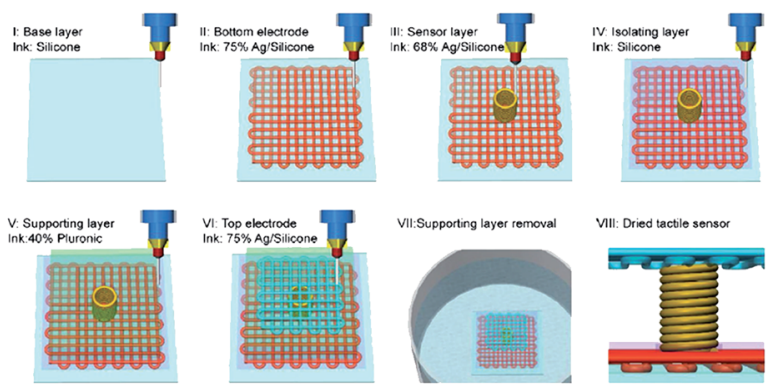

(b)
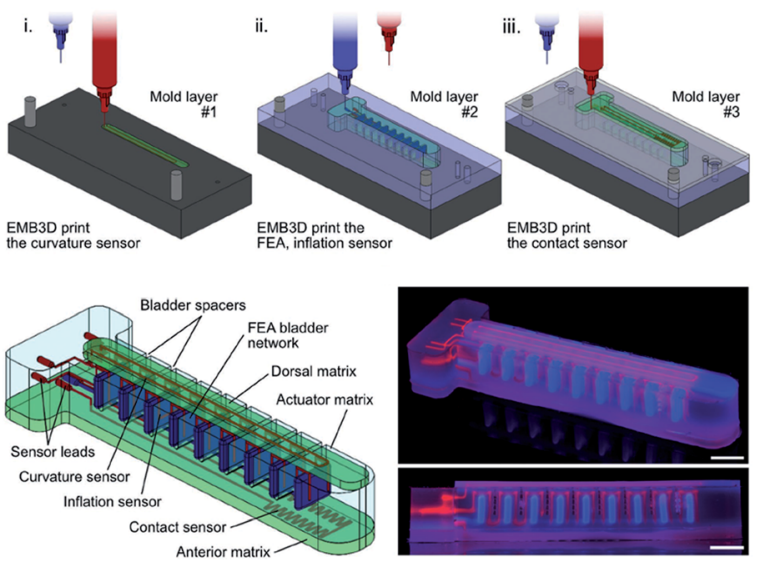

(c)

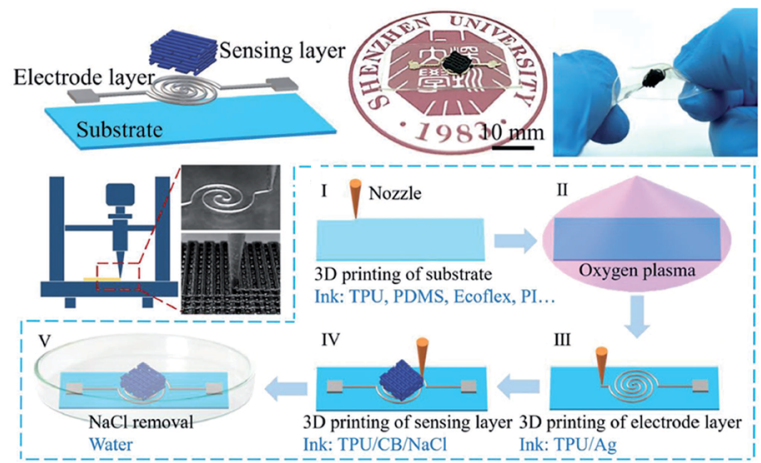

Fig. 5 Direct ink writing of multi-material sensor structures. (a) Fabrication of a multilayer tactile sensor using several cartridges and sequential printing; the device is manufactured using Ag/silicone inks with various solid loading fractions for each layer to create the functional components of the sensor (electrodes, sensor, isolation). To print overhanging features, a supporting hydrogel (Pluronic $($ ) layer is used and subsequently removed. Reproduced from ref. 83 with permission from John Wiley and Sons. (b) Somatosensitive actuator featuring multiple soft sensors produced via embedded 3D-printing using a combination of robocasting and moulds; each sensor is produced by printing directly into a matrix material in a mould. Three moulds, with three different matrices (dorsal, actuator, anterior - twopart platinum-cure silicone elastomers of different compositions) are used to host three different components (curvature, inflation, contact - 6 wt\% fumed silica in an ionic liquid EMIM-ES). Reproduced from ref. 59 with permission from John Wiley and Sons. (c) Stretchable piezoresistive sensor fabricated by step-by-step printing of a PDMS substrate (cured straight after printing using oxygen plasma treatment), electrode layer, and sensor layer. Reproduced from ref. 86 with permission from John Wiley and Sons. 
were formulated with poly(vinylidene-fluoride) PVDF, deposited onto pre-printed polydimethylsiloxane (PDMS) substrates and used as the cathode and anode respectively (Fig. 4d). A key step in this work is the formulation of a solid electrolyte using a polymer $N$-propyl- $N$-methyl pyrrolidinium bis(trifluoromethanesulfonyl)imide ( $\mathrm{Pyr}_{13}$ TFSI) and lithium bis(trifluoromethanesulfonyl)imide (LiTFSI) salt mixed on poly(vinylidene fluoride-co-hexafluoropropylene) (PVDF-coHFP) previously dissolved into $N$-methyl-2-pyrrolidone (NMP) solvent to then be filled with nanosized $\mathrm{TiO}_{2}$. They achieved a shear-thinning behaviour and very good wettability with the electrode substrates by elevated temperature DIW. A continuous, thin, and dense layer is formed between the porous electrolyte layer and the electrode, which effectively reduces the interfacial resistance of the solid-state battery. The interdigitated full battery was then sealed using polydimethylsiloxane (PDMS) gel under argon and showed an initial discharge capacity of $112 \mathrm{~mA} \mathrm{~h} \mathrm{~g}^{-1}$ at $16 \mathrm{~mA} \mathrm{~g}^{-1}$.

The latest work of Lewis et al. ${ }^{26}$ in this field shows a fully 3D printed and packaged LIB using DIW in which typical LIB manufacturing steps such as drying, electrolyte infilling, calendaring, clamping and heat sealing processes were eliminated by careful ink formulation together with encasing the custom-made 3D printer in an Ar-filled glove box and the use of UV light to cure the packaging seal (Fig. 4d). The formulation of inks to $3 \mathrm{D}$ print disparate materials without post-processing is a step forward and demonstrates the potential of this research area. Using biphasic semisolid carbon-ceramic mixtures with enhanced conductivity as thick electrodes and polymersceramic composites as separator and packaging they produced a fully 3D printed LIB that exhibited a ten-fold enhancement in areal capacity compared with their previous work. ${ }^{82}$ In a previous work, Sun et al. ${ }^{82}$ used the same technique to make a micro LIB battery with a more efficient approach to exploit the areal capacity by $3 \mathrm{D}$ printing interdigitated electrodes. The challenge was to seal the device in this configuration and they suggested to $3 \mathrm{D}$ print the packaging to solve this problem.

Beyond the field of energy storage there have been some demonstration of multi-cartridge printing in the area of sensors. Guo et al. ${ }^{\mathbf{3}}$ fabricated a 3D tactile sensor composed of 3 layers (top and bottom electrode and sensor layer) (Fig. 5c). The composition of the electrodes and the sensors were very similar (silver in silicone in different proportions) and the fabrication required to print a sacrificial layer to support the structure that is subsequently eliminated. This strategy has been previously employed to widen the range of structures that can be fabricated by DIW and requires the combination of several cartridges, one of them containing the support material that is eliminated during postprocessing. ${ }^{84,85}$

\section{Outlook, challenges and opportunities}

In this perspective, we have highlighted the potential of printing multi-material structures via direct ink writing (DIW). This is a promising manufacturing technology, that undoubtedly could contribute towards a sustainable future. We have reviewed potential and issues in formulation and processing for multi-material DIW in several engineering applications: energy storage devices, lightweight composites and sensors. From this review some key issues emerge:

\subsection{Formulation approaches}

One of the main challenges of multi-cartridge printing is to design and formulate the viscoplastic and self-healing soft inks that easily flow under shear and quickly recover upon deposition, which are required as inks to print different compounds. There are many strategies to formulate pastes for DIW. Researchers often look for flexible approaches that can be adapted to formulate printing inks with a wide range of materials. Beyond exhibiting the right rheology, it is usually preferable if the organic content of the ink is low (to limit de-binding problems) and its solid contents is high (to minimize dimensional changes after shaping). In the case of multi-material printing other requirements arise such as the need to minimise or avoid postprocessing in structures containing different materials and the adjustment of rheology to allow smooth switching of cartridges and the formation of strong, well connected interfaces.

\subsection{DIW systems}

Very often it is difficult to reach the right rheology to print parts that do not deform and retain their shape with inks that flow easily through the printing nozzle. In these cases, part consolidation during printing can be achieved using systems that incorporate a secondary process such as polymer curing or drying. When using multi-cartridge approaches there are still technical problems that should be addressed from a wider cross-disciplinary community. It is key to carry out fundamental studies combining fluid mechanics, rheology and physics to develop the methodologies needed to overcome some of the issues we face, for example when switching ink flow from one nozzle to the next one. This is sometimes approached as a trialerror adjustment of printing parameters. Often this is knowhow that is not openly shared or discussed in depth but that is critical for successful multi-material printing. Recently it has been shown how an alternative DIW system ${ }^{42}$ based on microfluidic printing heads enables very fast switching between materials and voxel by voxel fabrication to build soft-matter devices. The challenge now is to translate some of these concepts to the fabrication of hard, inorganic structures. This is an area where fundamental science will have to meet applied engineering, process control and machine learning. The development of systems able to monitor in situ printing variables (e.g. pressures and flows) and with the embedded "knowledge" to change them on the fly to correct for environmental fluctuations, or to adapt extrusion to create specific features, can bring multi-material printing closer to a commercial reality.

\subsection{Structural control}

One of the advantages of DIW for any application comes from the possibility to exert structural control simultaneously at 
multiple length scales from the micro to the macro levels. At the macro level, the technique can be used to implement some complex designs that may be difficult to access using other methodologies. For instance, DIW is particularly adept to the fabrication of light cellular structures. ${ }^{\mathbf{2 9 , 5 4 , 8 7}}$ However, extrusion technologies face challenges when printing bulk materials that require stacking of several layers as gas can be trapped between them. This problem can be solved by programming the right degree of overlap between printed lines and reducing the height between layers in order to deform to some extent the filament during printing to fill the gaps. ${ }^{29}$ In some cases, post-printing treatments such as cold or hot isostatic pressing or lamination can be applied. ${ }^{\mathbf{2 9 , 8 8}}$ These treatments will have an impact on the microstructure of the final materials. Another challenge in DIW is to print large overhangs or spans without sagging. This requires careful manipulation of formulation rheology but even when this is optimised, there are still some limits on the features that can be made using this technique. Sacrificial supports $^{84}$ or embedded printing in a gel ${ }^{89}$ have been used to expand the range of shapes that can be printed by DIW.

The examples reviewed in this paper show that extrusion opens some possibilities for microstructural manipulation (such as the generation of specific textures). However, further control is often needed and additives can be used to optimize the microstructure as it has been shown in flexible pressure sensors formed by PDMS microspheres and PDMS elastomer fibres coated with CNTs, where the incorporation of microspheres significantly enhanced performance. ${ }^{\mathbf{9 0}}$ Other approaches used sacrificial additions to create microporosity (for instance, to enhance tissue integration of implants and scaffolds) ${ }^{66,86}$ All these modifications and additions have to be done while maintaining the printability of the ink and retaining performance. An example is when fibre-reinforced epoxies are made printable by incorporating nano-clays. ${ }^{62}$ In this case there may be limits to the reinforcement content and it is important to ensure that the additions are not detrimental to the mechanical performance of the product.

\subsection{Upscaling}

Like for many other AM technologies, a key challenge for extrusion-based printing of structural materials is upscaling. Fabrication of large structures with fine details that require very small nozzles may require impractical time scales. One possibility is to modify the printing systems to combine printing heads with different resolutions. In addition, shaping often needs to be combined with subsequent treatments (e.g. drying, de-binding and sintering) while retaining dimensional accuracy and controlling the reactivity of the different components. Here, the combination of AM with novel fast densification technologies such as field assisted sintering can open new opportunities to build complex structures with phases that will be incompatible when using conventional heat treatments that require long firing times.

\subsection{Commercial impact}

The incentives to use 3D printing are cost-effectiveness (stock materials are often expensive), reduction of waste and ease of design implementation (printing of pre-designed structures). DIW has definitive potential on the easy and fast fabrication of prototypes for testing. However, when it comes to practical commercial application it will be necessary to take full advantage of its capabilities for the fabrication of complex structures that are not accessible by other processing technologies. It is unlikely that DIW will displace well established, mature technologies for mass production such as roll to roll fabrication of batteries but it may open new opportunities in the ad hoc design of devices in specific situations and the implementation of true $3 \mathrm{D}$ structures designed to optimize performance. In this respect, it should also be considered that the fabrication of three-dimensional devices also imposes constraints in subsequent packaging steps. An example is the field of lithium ion batteries where the potential to fully print high performance LIBs with nearly arbitrary form factors has been demonstrated. However, the papers often report printing of almost flat $2 \mathrm{D}$ configurations that could be easily accessible with other technologies in order to allow sealing of the thick electrodes that contain the active materials.

\subsection{Sustainability}

Conventional manufacturing processes can be very energy intensive and generate large amounts of waste. Additive manufacturing technologies, and in particular direct ink writing, can address some of these issues and play an important role in the development of more sustainable industries. They can be used to build parts on demand without moulds or machining, reducing consumption of raw materials and emissions. DIW presents the additional advantages of enabling the use of eco-friendly, often water-based, inks and low energy consumption as extrusion happens at room temperature using gantry robots. However, when looking at the development of cleaner, more sustainable manufacturing we should take into account that after shaping, consolidation usually requires energy-demanding treatments (e.g. drying or sintering). It is necessary to take a holistic approach in which additive manufacturing can play a key part. As shown in this paper, in the case of DIW this could involve the minimization of postprinting procedures, the integration of shaping and consolidation in a single step or the combination with new, more efficient sintering technologies (e.g. field assisted or cold sintering).

Multi-material additive manufacturing will also contribute to a more sustainable society by opening new possibilities on the fabrication of structures and devices. Some of the applications have been reviewed here and include ultralight structures, longer-lasting implants and tissue engineering scaffolds with improved bio-integration, wearable sensors or novel devices for energy storage and generation. All these technologies have the potential to change the way we store and consume energy and to provide longer lasting, more efficient solutions for a wide range of fields from health care to construction and transportation. Here DIW has an advantage over other additive manufacturing technologies in that, despite the technical challenges, the integration of different materials in a single processing step is 
conceptually straightforward. As we have shown, these challenges are being currently addressed and numerous solutions are being developed. With these solutions DIW enables the rapid fabrication and testing of new design concepts, accelerating discovery in a way that is not easily accessible with other techniques. Some of these concepts may be commercially implemented using other technologies but DIW could also be play a role in manufacturing. It already allows the ad hoc fabrication of devices for specific applications in relatively small batches (e.g. custom designed scaffolds or sensors). However, the issues of upscaling device size and reducing production times to enable large batches are still barriers for mass implementation. Solving these problems will open new opportunities to create a more sustainable economy.

The challenges are multiple but multi-material direct ink writing offers a unique space for collaborative research. This collaboration should include not only those working on processing or machine design but also the end users. Their needs can offer guidelines for technique development. It is often not only about enhancing printing precision or fabrication speeds, but also building systems that are reliable and easy to use in a production environment. The outcomes of this research will have an extremely broad impact and support progress in many applications from health, chemistry and materials science to engineering and entertainment thus the establishment of wider multi-disciplinary collaborations would be hugely beneficial.

\section{Conflicts of interest}

There are no conflicts to declare.

\section{Acknowledgements}

ES and IE would like to acknowledge the EPSRC Future Manufacturing Hub in Manufacture using Advanced Powder Processes (MAPP) EP/P006566/1.

\section{Notes and references}

1 U. Nations, The 2030 Agenda for Sustainable Development, United Nations Department of Economic and Social Affairs, 2015.

2 W. Gao, Y. Zhang, D. Ramanujan, K. Ramani, Y. Chen, C. B. Williams, C. C. L. Wang, Y. C. Shin, S. Zhang and P. D. Zavattieri, Comput.-Aided Des., 2015, 69, 65-89.

3 A. Zhakeyev, P. Wang, L. Zhang, W. Shu, H. Wang and J. Xuan, Adv. Sci., 2017, 4, 1700187.

4 S. C. Ligon, R. Liska, J. Stampfl, M. Gurr and R. Mulhaupt, Chem. Rev., 2017, 117, 10212-10290.

5 S. C. Daminabo, S. Goel, S. A. Grammatikos, H. Y. Nezhad and V. K. Thakur, Mater. Today Chem., 2020, 16, 100248.

6 D. D. Gu, W. Meiners, K. Wissenbach and R. Poprawe, Int. Mater. Rev., 2013, 57, 133-164.

7 T. DebRoy, H. L. Wei, J. S. Zuback, T. Mukherjee, J. W. Elmer, J. O. Milewski, A. M. Beese, A. Wilson-Heid, A. De and W. Zhang, Prog. Mater. Sci., 2018, 92, 112-224.
8 S. Das, D. L. Bourell and S. S. Babu, MRS Bull., 2016, 41, 729741.

9 Z. Chen, Z. Li, J. Li, C. Liu, C. Lao, Y. Fu, C. Liu, Y. Li, P. Wang and Y. He, J. Eur. Ceram. Soc., 2019, 39, 661-687.

$10 \mathrm{H}$. Li and J. Liang, Adv. Mater., 2020, 32, e1805864.

11 C. Zhu, T. Liu, F. Qian, W. Chen, S. Chandrasekaran, B. Yao, Y. Song, E. B. Duoss, J. D. Kuntz, C. M. Spadaccini, M. A. Worsley and Y. Li, Nano Today, 2017, 15, 107-120.

12 P. Chang, H. Mei, S. Zhou, K. G. Dassios and L. Cheng, J. Mater. Chem. A, 2019, 7, 4230-4258.

13 M. P. Browne, E. Redondo and M. Pumera, Chem. Rev., 2020, 120(5), 2783-2810.

14 F. Zhang, M. Wei, V. V. Viswanathan, B. Swart, Y. Shao, G. Wu and C. Zhou, Nano Energy, 2017, 40, 418-431.

15 M. Cheng, R. Deivanayagam and R. Shahbazian-Yassar, Batteries Supercaps, 2020, 3, 130-146.

16 Y. Z. Zhang, Y. Wang, T. Cheng, L. Q. Yao, X. Li, W. Y. Lai and W. Huang, Chem. Soc. Rev., 2019, 48, 3229-3264.

17 A. Ambrosi and M. Pumera, Chem. Soc. Rev., 2016, 45, 27402755.

18 X. Tian, J. Jin, S. Yuan, C. K. Chua, S. B. Tor and K. Zhou, Adv. Energy Mater., 2017, 7, 1700127.

19 T. S. Arthur, D. J. Bates, N. Cirigliano, D. C. Johnson, P. Malati, J. M. Mosby, E. Perre, M. T. Rawls, A. L. Prieto and B. Dunn, MRS Bull., 2011, 36, 523-531.

20 E. MacDonald and R. Wicker, Science, 2016, 353(6307), aaf2093.

21 A. Lee, A. Hudson, D. Shiwarski, J. Tashman, T. Hinton, S. Yerneni, J. Bliley, P. Campbell and A. Feinberg, Science, 2019, 365, 482-487.

22 S. V. Murphy and A. Atala, Nat. Biotechnol., 2014, 32, 773.

23 M. S. Mannoor, Z. Jiang, T. James, Y. L. Kong, K. A. Malatesta, W. O. Soboyejo, N. Verma, D. H. Gracias and M. C. McAlpine, Nano Lett., 2013, 13, 2634-2639.

24 A. J. Blake, R. R. Kohlmeyer, J. O. Hardin, E. A. Carmona, B. Maruyama, J. D. Berrigan, H. Huang and M. F. Durstock, Adv. Energy Mater., 2017, 7(14), 1602920.

25 M. Wei, F. Zhang, W. Wang, P. Alexandridis, C. Zhou and G. Wu, J. Power Sources, 2017, 354, 134-147.

26 T. S. Wei, B. Y. Ahn, J. Grotto and J. A. Lewis, Adv. Mater., 2018, 30, e1703027.

27 V. G. Rocha, E. Garcia-Tunon, C. Botas, F. Markoulidis, E. Feilden, E. D'Elia, N. Ni, M. Shaffer and E. Saiz, ACS Appl. Mater. Interfaces, 2017, 9, 37136-37145.

28 B. G. Compton and J. A. Lewis, Adv. Mater., 2014, 26, 59305935.

29 E. Feilden, C. Ferraro, Q. Zhang, E. Garcia-Tunon, E. D'Elia, F. Giuliani, L. Vandeperre and E. Saiz, Sci. Rep., 2017, 7, 13759.

30 J. T. Muth, D. M. Vogt, R. L. Truby, Y. Mengüç, D. B. Kolesky, R. J. Wood and J. A. Lewis, Adv. Mater., 2014, 26, 6307-6312.

31 M. Wehner, R. L. Truby, D. J. Fitzgerald, B. Mosadegh, G. M. Whitesides, J. A. Lewis and R. J. Wood, Nature, 2016, 536, 451-455.

32 J. E. Smay, J. Cesarano and J. A. Lewis, Langmuir, 2002, 18, 5429-5437. 
33 J. A. Lewis, J. E. Smay, J. Stuecker and J. Cesarano, J. Am. Ceram. Soc., 2006, 89, 3599-3609.

34 E. Garcia-Tunon, E. Feilden, H. Zheng, E. D'Elia, A. Leong and E. Saiz, ACS Appl. Mater. Interfaces, 2017, 9, 3297732989.

35 A. Corker, H. C. Ng, R. J. Poole and E. Garcia-Tunon, Soft Matter, 2019, 15, 1444-1456.

36 R. L. Truby and J. A. Lewis, Nature, 2016, 540, 371-378.

37 A. Z. Nelson and R. H. Ewoldt, Soft Matter, 2017, 13, 75787594.

38 M. Greaves, S. Barg and M. A. Bissett, Batteries Supercaps, 2020, 3, 214.

39 P. T. Smith, A. Basu, A. Saha and A. Nelson, Polymer, 2018, 152, 42-50.

40 A. M'barki, L. Bocquet and A. Stevenson, Sci. Rep., 2017, 7, 110.

41 J. O. Hardin, T. J. Ober, A. D. Valentine and J. A. Lewis, Adv. Mater., 2015, 27, 3279-3284.

42 M. A. Skylar-Scott, J. Mueller, C. W. Visser and J. A. Lewis, Nature, 2019, 575, 330-335.

43 T. J. Ober, D. Foresti and J. A. Lewis, Proc. Natl. Acad. Sci. U. S. A., 2015, 112, 12293-12298.

44 J. M. Ortega, M. Golobic, J. D. Sain, J. M. Lenhardt, A. S. Wu, S. E. Fisher, L. X. Perez Perez, A. W. Jaycox, J. E. Smay and E. B. Duoss, Adv. Mater. Technol., 2019, 4, 1800717.

45 N. A. Dudukovic, L. L. Wong, D. T. Nguyen, J. F. Destino, T. D. Yee, F. J. Ryerson, T. Suratwala, E. B. Duoss and R. Dylla-Spears, ACS Appl. Nano Mater., 2018, 1, 4038-4044.

46 A. Nadernezhad, N. Khani, G. A. Skvortsov, B. Toprakhisar, E. Bakirci, Y. Menceloglu, S. Unal and B. Koc, Sci. Rep., 2016, 6, 1-12.

47 X. Li, J. M. Zhang, X. Yi, Z. Huang, P. Lv and H. Duan, Adv. Sci., 2019, 6, 1800730.

48 S. Nesaei, Y. Song, Y. Wang, X. Ruan, D. Du, A. Gozen and Y. Lin, Anal. Chim. Acta, 2018, 1043, 142-149.

49 K. J. Chen, X. Kuang, V. Li, G. Z. Kang and H. J. Qi, Soft Matter, 2018, 14, 1879-1886.

50 M. Cheng, Y. Jiang, W. Yao, Y. Yuan, R. Deivanayagam, T. Foroozan, Z. Huang, B. Song, R. Rojaee, T. Shokuhfar, Y. Pan, J. Lu and R. Shahbazian-Yassar, Adv. Mater., 2018, 30, e1800615.

51 L. Fieber, J. D. Evans, C. Huang and P. S. Grant, Addit. Manuf., 2019, 28, 344-353.

52 K. Chen, L. Zhang, X. Kuang, V. Li, M. Lei, G. Kang, Z. L. Wang and H. J. Qi, Adv. Funct. Mater., 2019, 29, 1903568.

53 H. Zhu, Y. D. Kim and D. De Kee, J. Non-Newtonian Fluid Mech., 2005, 129, 177-181.

54 G. Franchin, L. Wahl and P. Colombo, J. Am. Ceram. Soc., 2017, 100, 4397-4401.

55 B. Dorj, J. E. Won, J. H. Kim, S. J. Choi, U. S. Shin and H. W. Kim, J. Biomed. Mater. Res., Part A, 2013, 101, 16701681.

56 X. You, J. Yang, M. Wang, J. Hu, Y. Ding, X. Zhang and S. Dong, 2D Materials, 2019, 7(1), 015025.

57 M. Abshirini, M. Charara, P. Marashizadeh, M. C. Saha, M. C. Altan and Y. Liu, Appl. Nanosci., 2019, 9, 2071-2083.
58 S. Kim, J. Oh, D. Jeong and J. Bae, ACS Appl. Mater. Interfaces, 2019, 11, 20557-20565.

59 R. L. Truby, M. Wehner, A. K. Grosskopf, D. M. Vogt, S. G. M. Uzel, R. J. Wood and J. A. Lewis, Adv. Mater., 2018, 30, e1706383.

60 F. Kim, B. Kwon, Y. Eom, J. E. Lee, S. Park, S. Jo, S. H. Park, B.-S. Kim, H. J. Im, M. H. Lee, T. S. Min, K. T. Kim, H. G. Chae, W. P. King and J. S. Son, Nat. Energy, 2018, 3, 301-309.

61 C. S. Marchi, M. Kouzeli, R. Rao, J. A. Lewis and D. C. Dunand, Scr. Mater., 2003, 49, 861-866.

62 N. S. Hmeidat, J. W. Kemp and B. G. Compton, Compos. Sci. Technol., 2018, 160, 9-20.

63 D. Kokkinis, M. Schaffner and A. R. Studart, Nat. Commun., 2015, 6, 8643.

64 T. J. Wallin, J. Pikul and R. F. Shepherd, Nat. Rev. Mater., 2018, 3, 84-100.

65 P. Jiang, Z. Ji, X. Zhang, Z. Liu and X. Wang, Progress in Additive Manufacturing, 2017, 3, 65-86.

66 Y. Zhang, G. Shi, J. Qin, S. E. Lowe, S. Zhang, H. Zhao and Y. L. Zhong, ACS Appl. Electron. Mater., 2019, 1, 1718-1734.

67 S. Tibbits, Archit. Des., 2014, 84, 116-121.

68 A. S. Gladman, E. A. Matsumoto, R. G. Nuzzo, L. Mahadevan and J. A. Lewis, Nat. Mater., 2016, 15, 413-418.

69 Y. Kim, H. Yuk, R. Zhao, S. A. Chester and X. Zhao, Nature, 2018, 558, 274-279.

70 X. Wan, H. Wei, F. Zhang, Y. Liu and J. Leng, J. Appl. Polym. Sci., 2019, 136(44), 48177.

71 M. Grossman, F. Bouville, K. Masania and A. R. Studart, Proc. Natl. Acad. Sci. U. S. A., 2018, 115, 12698-12703.

72 T. P. Niebel, D. Carnelli, M. R. Binelli, R. Libanori and A. R. Studart, J. Mech. Behav. Biomed. Mater., 2016, 60, 367377.

73 J. E. Smay, B. Tuttle and J. C. Iii, in Piezoelectric and Acoustic Materials for Transducer Applications, Springer, US, 2008, pp. 305-318, DOI: 10.1007/978-0-387-76540-2_15.

74 A. M. Golobic, M. D. Durban, S. E. Fisher, M. D. Grapes, J. M. Ortega, C. M. Spadaccini, E. B. Duoss, A. E. Gash and K. T. Sullivan, Adv. Eng. Mater., 2019, 21(8), 1900147.

75 Y. L. Xia, Z. L. Lu, A. J. W. Cao, K. Miao, J. Li and D. C. Li, Scr. Mater., 2019, 165, 84-88.

76 Z. W. Fu, M. Freihart, T. Schlordt, T. Fey, T. Kraft, P. Greil and N. Travitzky, Rapid Prototyp. J., 2017, 23, 423-433.

77 J. Mueller, J. R. Raney, K. Shea and J. A. Lewis, Adv. Mater., 2018, 30, 1705001.

78 C. Paredes, F. J. Martinez-Vazquez, A. Pajares and P. Miranda, Ceram. Int., 2019, 45, 19572-19576.

79 K. Shen, J. Ding and S. Yang, Adv. Energy Mater., 2018, 8, 1800408.

80 E. Malone, M. Berry and H. Lipson, Rapid Prototyp. J., 2008, 14, 128-140.

81 L. J. Deiner, C. A. G. Bezerra, T. G. Howell and A. S. Powell, Adv. Eng. Mater., 2019, 21(11), 1900737.

82 K. Sun, T. S. Wei, B. Y. Ahn, J. Y. Seo, S. J. Dillon and J. A. Lewis, Adv. Mater., 2013, 25, 4539-4543.

83 S. Z. Guo, K. Qiu, F. Meng, S. H. Park and M. C. McAlpine, Adv. Mater., 2017, 29, 1701218. 
84 F. J. Martinez-Vazquez, A. Pajares and P. Miranda, J. Eur. Ceram. Soc., 2018, 38, 2247-2250.

85 J. A. Lewis, Adv. Funct. Mater., 2006, 16, 2193-2204.

86 Z. Wang, X. Guan, H. Huang, H. Wang, W. Lin and Z. Peng, Adv. Funct. Mater., 2019, 29(11), 1807569.

87 A. M. Stanciuc, C. M. Sprecher, J. Adrien, L. I. Roiban, M. Alini, L. Gremillard and M. Peroglio, J. Eur. Ceram. Soc., 2018, 38, 845-853.
88 I. K. Jones, Z. M. Seeley, N. J. Cherepy, E. B. Duoss and S. A. Payne, Opt. Mater., 2018, 75, 19-25.

89 A. K. Grosskopf, R. L. Truby, H. Kim, A. Perazzo, J. A. Lewis and H. A. Stone, ACS Appl. Mater. Interfaces, 2018, 10, 23353-23361.

90 Y. Gao, M. Xu, G. Yu, J. Tan and F. Xuan, Sens. Actuators, A, 2019, 299, 111625. 